\title{
A Novel Behavorial Model Of Power Amplifier Based On A Dynamic Envelope Gain Approach For The System Level Simulation And Design
}

\author{
C.Maziere*, T.Reveyrand**, S.Mons*, D.Barataud*, J.M.Nebus* , R.Quere*, \\ A.Mallet**, L.Lapierre** And J.Sombrin** \\ * IRCOM - UMR CNRS 6615 - 123 Avenue Albert-Thomas 87060 LIMOGES Cedex \\ ** CNES - 18 Avenue Edouard-Belin 31055 TOULOUSE Cedex \\ e-mail : maziere@ircom.unilim.fr
}

\begin{abstract}
This paper presents a novel and non quasi static behavioral ( black box ) model of power amplifiers. It takes into account nonlinear memory effects for an accurate prediction of signal distortions and power budget in communication systems. The model topology and the model extraction procedure are described. The model proposed in this paper can be derived either from envelope simulation results of a PA circuit design or from time domain envelope measurements of a PA driven by modulated stimuli. Examples of new measurements (ie : dynamic AM/AM conversion characteristics) highlight nonlinear memory of SSPAs . The behavioral model proposed is very well suited for an efficient implementation in system level software packages because it consists of an extension of the complex envelope gain function. The proposed complex gain which depends on the input envelope and its first time derivatives provides a great enhancement as compared to the memoryless static complex gain derived from classical AM/AM and AM/PM conversion characteristics.
\end{abstract}

\section{INTRODUCTION}

The analysis and the simulation of communication systems in order to optimally design the system architecture and the equipment sizing is very important to reach the best performances at the lowest cost.

This task requires accurate behavioral (black box) models of power amplifiers which are critical elements in the power budget of a communication link.

Inaccurate predictions of linearity and power efficiency issues of PAs at the system level lead generally to oversized design solutions which are critical to the cost versus performance trade off. Inaccuracies are well known to be quite important when memory less (quasi static) behavioural models of PAs are assumed.

Dynamic nonlinear effects (memory effects) are due to thermal aspects engaged in active cells of PAs as well as low frequency currents/voltages at the envelope frequency of RF modulated signals which are present in biasing circuits of PAs. They strongly impact the linearity and efficiency features. These quite complicated effects need to be handle by new behavioral models for an enhancement of the system level analysis, simulation and design.

The first part of the paper focuses on an original way of probing and demonstrating the presence of nonlinear memory that exhibit PAs. It consists in the plots of dynamic AM/AM characteristics obtained for different modulated test signals. This has been done with envelope simulations of a PA circuit design $(20 \mathrm{GHz}-2$ Watt- $20 \mathrm{~dB}$ gain). On the other hand, it is also illustrated through new calibrated time domain envelope measurements of a $\mathrm{L}$ Band-4 Watt-30 dB power gain SSPA.

Then in a second part, the proposed behavioral modeling approach is presented. It consists of a dynamic envelope gain function which can be identified either from envelope simulation results or envelope measurements.

In a third and last part the enhancement provided by the novel approach as compared to memoryless approach is highlighted.

\section{DYNAMIC ENVELOPE GAIN BEHAVIOUR OF SSPA's}

PAs exhibit nonlinear memory effects due to both short term and long term time constants inherent to RF matching circuits, biasing circuits and thermal aspects present in active cells[1]. Consequently the output signal of a PA can not be accurately determined by using the static AM/AM and AM/PM conversion characteristics because this assume a purely static behavior (i.e.: the output at a time instant depends only on the input at the same time instant). It is of prime importance to characterize memory effects in nonlinear devices because these dynamic effects impact signal distortions (linearity) as well as power efficiency performances.

Analyses and computations of signal distortions and power performances at the system level make use of the 
time domain envelope formalism : analytical signals associated to real RF signals at both ports of $50 \mathrm{ohms}$ matched PAs.

Consider $x(t)=A(t) \cdot \cos \left(\omega_{0} \cdot t+\varphi(t)\right)$ and $y(t)=B(t) \cdot \cos \left(\omega_{0} \cdot t+\theta(t)\right)$ respectively the input and output useful signals, $\tilde{X}(t)=A(t) \cdot e^{j \cdot \varphi(t)}$ and $\tilde{Y}(t)=B(t) \cdot e^{j \cdot \theta(t)}$ are the corresponding complex envelopes. One of the most difficult problem is to define a nonlinear function linking $\tilde{Y}(t)$ with $\tilde{X}(t)$ and an efficient approach for the identification of this function in order to take into account nonlinear memory effects.

For that purpose, envelope simulations or envelope measurements are necessary. Simulations of nonlinear PA circuits driven by modulated signals are now currently carried out using envelope simulation tools of CAD packages. Therefore, considering a PA circuit design (Fig.1), $\tilde{Y}(t)$ can be simulated and analysed for several different $\tilde{X}(t)$. A nice and original way to highlight the presence of nonlinear memory effects is to plot the instantaneous magnitude of $\tilde{Y}(t)$ versus the instantaneous magnitude of $\tilde{X}(t)$.

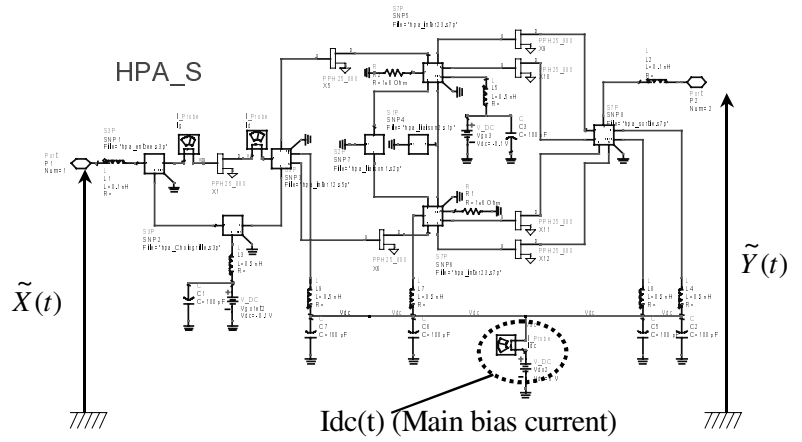

Fig. 1. 2W-20GHz-3 stage-PA circuit

Two tone signals with various power level and tone spacing are quite simple and efficient test signals for that. Fig. 2 shows this kind of simulation results.

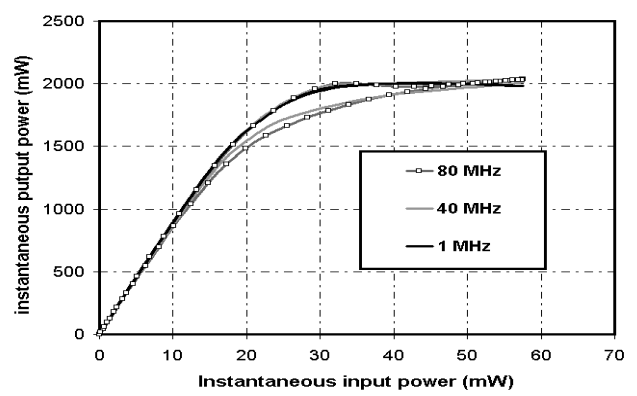

Fig. 2. Dynamic AM/AM simulations for two tone stimuli with different tone spacing.

The tone spacing varies from $1 \mathrm{MHz}$ to $80 \mathrm{MHz}$. These curves indicate the presence of nonlinear memory effects at high power level for wide tone spacing. An hysteresis is observed on the curve representing the output/input envelope trajectory. These dynamic AM/AM characteristics are then quite different from the conventional static AM/AM characteristics currently recorded with $\mathrm{CW}$ test signals.

In this case, it has been identified to be due to a non ideal impedance profile of biasing circuits at a frequency higher than $40 \mathrm{MHz}$.

The presence of these low frequency memory effects can also be shown by plotting the shape of the bias current $\operatorname{Idc}(\mathrm{t})$ of $3^{\text {rd }}$ power stage:

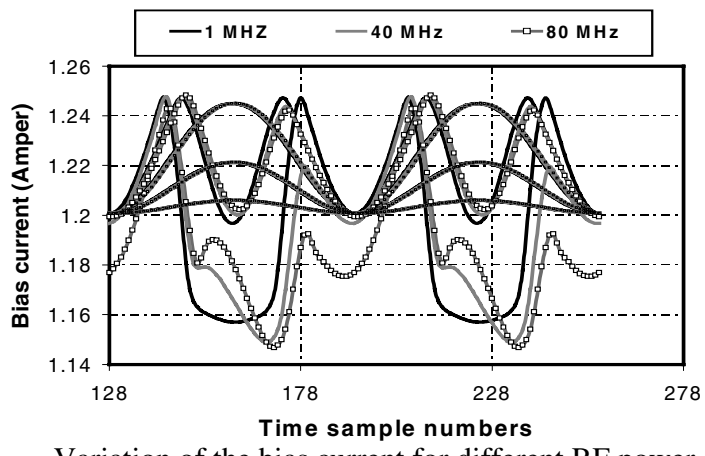

Fig. 3. Variation of the bias current for different RF power level and tone spacing ranging from $1 \mathrm{MHz}$ to $80 \mathrm{MHz}$

It is also very important to highlight and characterize such phenomena with measurements[2][3]. For that we have developed a calibrated set-up for the measurements of time domain envelopes of modulated RF carriers at the input and the output of amplifiers under test[4][5]. The block diagram is shown in Fig. 4.

An Arbitrary waveform generator is used to synthesize base band envelope stimuli. Up conversion to the RF domain is achieved by using an I/Q modulator. The RF signal at the output of a nonlinear DUT is down converted thanks to an I/Q demodulator to obtain base band signals measured by a sampling scope. A calibration procedure is applied to get error corrected envelopes at the DUT reference planes.

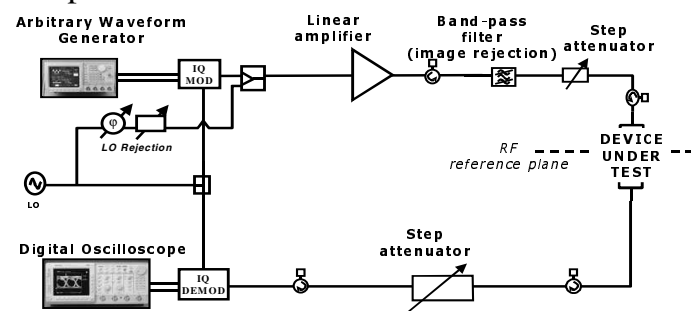

Fig. 4. The measurement setup block diagram

Measurements of a 4 Watt HFET PA at L Band have been performed for different test signals (two tone, QPSK, multi tone). Dynamic AM/AM measurements are shown in Fig. 5: 


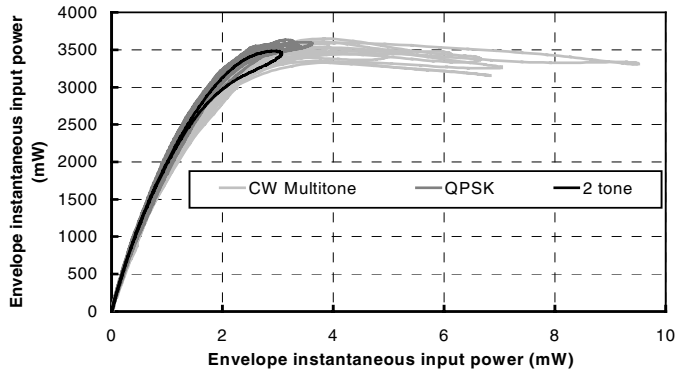

Fig. 5. Dynamic AM/AM measurements for different kind of modulation

These measurement results highlight the presence of nonlinear memory effects due to both thermal aspects and low frequency signal (at the envelope frequency) present in the internal biasing circuits of the PA when this one operates in a nonlinear regime. Such measurements which are novel and demonstrative enforce the necessity to take into account memory effects in the behavioral modeling of PAs for system level analysis, simulation and design.

\section{A New BeHAVORIAl MODELING APPROACH}

The idea consists in establishing a relationship between the input and output envelopes following an explicit formulation :

$$
\tilde{y}(t)=f_{N L}\left(\tilde{x}(t), \frac{\partial \tilde{x}(t)}{\partial t}, \frac{\partial^{2} \tilde{x}(t)}{\partial t^{2}}, \frac{\partial^{3} \tilde{x}(t)}{\partial t^{3}}, \ldots ., \frac{\partial^{n} \tilde{x}(t)}{\partial t^{n}}\right)
$$

Assuming a first order approximation, one can write :

$$
\tilde{y}(t)=f_{N L}\left(\tilde{x}(t), \frac{\partial \tilde{x}}{\partial t}\right)=f_{N L}\left(A(t), \frac{\partial A(t)}{\partial t}, \varphi(t), \frac{\partial \varphi(t)}{\partial t}\right)
$$

Due to the time invariance $\tilde{G}_{N L}=\frac{\tilde{y}(t)}{\widetilde{x}(t)}$ does not depend on $\varphi(t)$ and can be expressed as:

$$
\begin{aligned}
& \operatorname{Re}\{\tilde{G}(t)\}=h_{1 N L}\left(A(t), \frac{\partial A(t)}{\partial t}, \frac{d \varphi(t)}{d t}\right) \\
& \operatorname{Im}\{\tilde{G}(t)\}=h_{2 N L}\left(A(t), \frac{\partial A(t)}{\partial t}, \frac{d \varphi(t)}{d t}\right)
\end{aligned}
$$

The first time derivatives $\frac{\partial A(t)}{\partial t}, \frac{\partial \varphi(t)}{\partial t}$ are key parameters for the characterization of nonlinear slow dynamics (group delay, thermal dependence, spurious modulation of bias points). Indeed, the response of the amplifier is often different whereas the same instanteous power level is applied to the system if the derivative $\frac{\partial A(t)}{\partial t}$ is different.

This leads to output envelope assymetry which is another "visual" criteria for memory effects (Fig. 6). In the same manner, the response varies with the instantaneous envelope frequency $\left(\Omega=\frac{\partial \varphi(t)}{\partial t}\right)$.

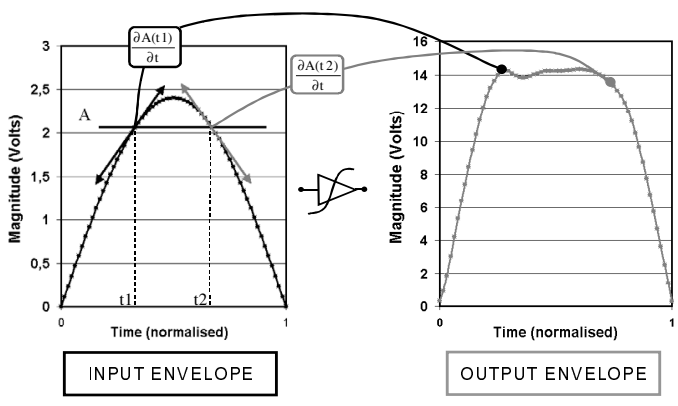

Fig. 6. Description of memory effect (Amplitude)

The variations of $A(t), \frac{\partial A(t)}{\partial t}, \frac{\partial \varphi(t)}{\partial t}$ can be achieved in a quite straightforward manner by scanning the $3 \mathrm{D}$ space with test signals like two tone stimuli with various power, tone spacing and frequency position $(\Omega)$ :

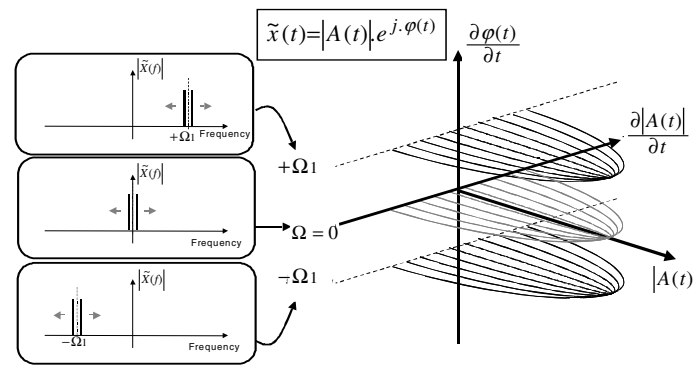

Fig. 7. Definition domain of the new Model

These data can be collected either by envelope transient simulation or time domain measurement. Then, both $\tilde{G}(t)$ and $I d c(t)$ can be fitted by Neural Networks[6]:

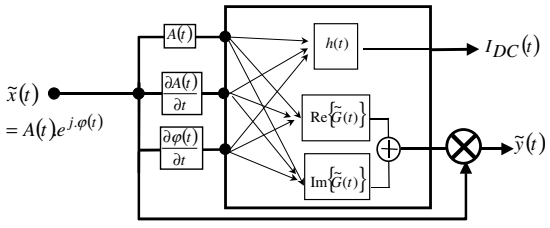

Fig. 8. Behavioral model topology

Thus, both the output signal and the DC consumption can be predicted for different input signals. As a consequence, PAE and linearity performances can be computed.

\section{Demonstrative Capabilities Of The New BEHAVORIAL MODEL}

\section{A. Behavorial Model derivated from Circuit simulation}

Circuit Simulation of a $20 \mathrm{GHz}-2 \mathrm{~W}$ PA were performed for two tone stimuli (tone spacing varied from $1 \mathrm{MHZ}$ to $160 \mathrm{MHz}$ ). The input power has been swept from low to high power level (5 dB gain compression). These data 
were used for the model training procedure. Then, IM3 values have been predicted by using our new model, a purely static model and compared to circuit simulations.

Obviously for this comparison, the test signals are different from the ones used for the training procedure of the Neural Network. Fig. 9a shows the results:

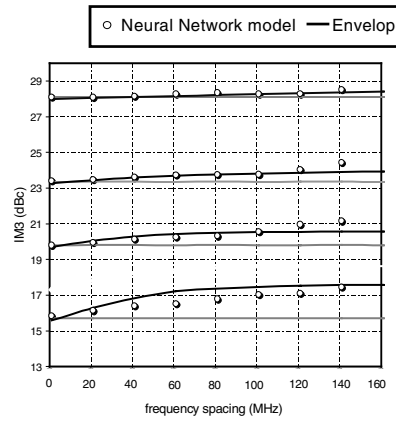

(a)

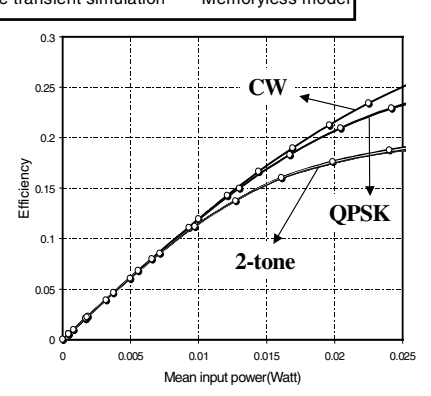

(b)
Fig. 9. IM3 versus Power and tone spacing and efficiency given by New Model/circuit simulation

Such results emphasize the enhancement given by the dynamic gain approach (IM3 variations with tone spacing are predicted).

We also show in Fig. 9b efficiency performances derived from circuit simulation and dynamic Model prediction (system level simulation). This was done for different stimuli: CW, two tone signal and QPSK modulation (the Bit rate was fixed at $20 \mathrm{MB} / \mathrm{s}$ and a rolloff of 0.35 was taken). Good predictions are obtained for signals which are different from the ones used for the model extraction.

\section{B. Behavorial Model derivated from Time domain envelope measurement}

The same kind of work was carried out using measurement based data. A 4W L Band PA was measured using the calibrated time domain envelope set up mentioned in II. A multitone (64 tones) test signal was used to build a data file for the training session of the neural network[5]. It's a quite attractive and convenient test signal to get a suitable set of measurement points covering uniformly the definition domain ( $A(t), \frac{\partial A(t)}{\partial t}, \frac{\partial \varphi(t)}{\partial t}$ ) for the model extraction purpose. Then the behavorial model was used for IM3 predictions.

Dynamic behavorial model predictions, static prediction (memoryless AM/AM AM/PM) and measurements are compared in Fig. 10. These curves really demonstrate here the significant enhancement provided by our dynamic behavioral modeling approach for the predictions of nonlinear envelope distortions caused by microwave solid state power devices.

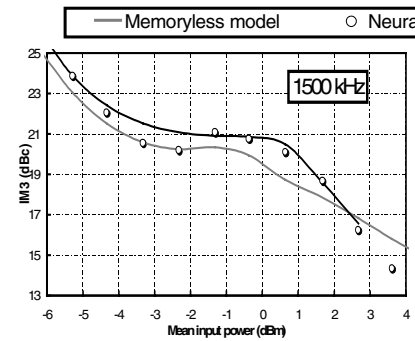

(a)

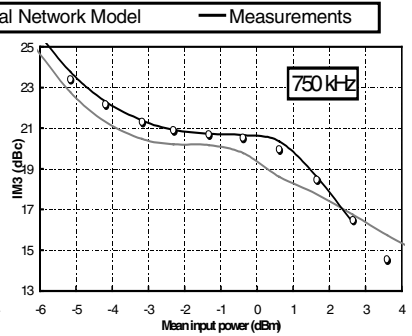

(b)
Fig. 10. IM3 of New Model / Measurement / Memoryless Model

\section{CONCLUSION}

This paper has presented a new calibrated time domain envelope measurements and a new behavorial modeling technique. This new approach has several advantages: It takes into account nonlinear memory effects and the model extraction can be based on simulation or experimental data in easy ways. The model topology enables both a good prediction of envelope distorsion and DC consumption for different kind of modulations. Its simple formulation and the use of neural networks enables an easy implementation in system level simulator.

\section{ACKNOWLEDGEMENT}

The authors wish to acknowledge J.L Cazaux, Nicolas Le Gallou, Alcatel Space Industries for providing the HFET amplifier.

\section{REFERENCES}

[1] N. Le Gallou, \&al, "Analysis of low frequency memory and influence on solid state HPA intermodulation characteristics", IEEE MTT-S Digest, Phoenix, vol. 2, pp.979-982, May 2001

[2] A.A. Moulthrop, \&al, "A new time domain measurement technique for microwave devices", International Microwave Symposium Digest 1998, pp.945-948, 1998

[3] W. Bösch, G. Gatti, "Measurement and simulation of memory effects in predistortion linearizers", IEEE Transactions on Microwave Theory and Techniques, vol. 37, $\mathrm{n}^{\circ} 12$, December 1989, pp 1885-1890.

[4] A. Mallet, \&al, "A new satellite repeater amplifier characterization system for large bandwidth NPR and modulated signals measurements", IEEE MTT-S Digest, Seattle, Vol. 3, pp 2245-2248, June 2002-06-27

[5] T. Reveyrand, \&al, "A calibrated time-domain envelope measurement system for the behavorial modelling of power amplifier", European Microwave Week, GaAS conference, oct 2002

[6] Y. Harkouss, \&al, "The use of artificial neural networks in nonlinear microwave devices and circuits modeling : An application to telecommunication system design (invited article)", International Journal of $R F$ and Microwave Computer-Aided Engineering, vol. 9, Issue 3, pp. 198-215, May 1999. 